\title{
Modelo de innovación educativa según las experiencias de docentes y estudiantes universitarios
}

\author{
Model of educational innovation according to the experiences of teachers and \\ university students
}

Modelo de inovação educacional de acordo com as experiências de professores e estudantes universitários

Martha Alejandrina Zavala-Guirado Instituto Tecnológico de Sonora, Unidad Guaymas, México

martha.zavala@itson.edu.mx https://orcid.org/0000-0001-9177-0411

Isolina González-Castro Instituto Tecnológico de Sonora, Unidad Guaymas, México igonzalez@itson.edu.mx https://orcid.org/0000-0002-7265-6725

Mario Alberto Vázquez-García Instituto Tecnológico de Sonora, Unidad Guaymas, México mvazquez@itson.edu.mx https://orcid.org/0000-0002-6490-838X

\section{Resumen}

El objetivo del presente estudio fue identificar las prácticas innovadoras que utilizan los docentes para desarrollar el proceso de enseñanza-aprendizaje en el aula con el fin de desarrollar una teoría fundamentada sobre la innovación educativa que existe en la práctica docente. En la investigación participaron 17 docentes y 25 estudiantes de una universidad pública del estado de Sonora, México. Los resultados obtenidos revelan un modelo didáctico constructivista con un enfoque socioformativo en las estrategias didácticas y de evaluación formativa, así como funciones y roles docentes humanistas basados en el respeto, la empatía y la confianza, lo cual se apoya con el empleo de medios 


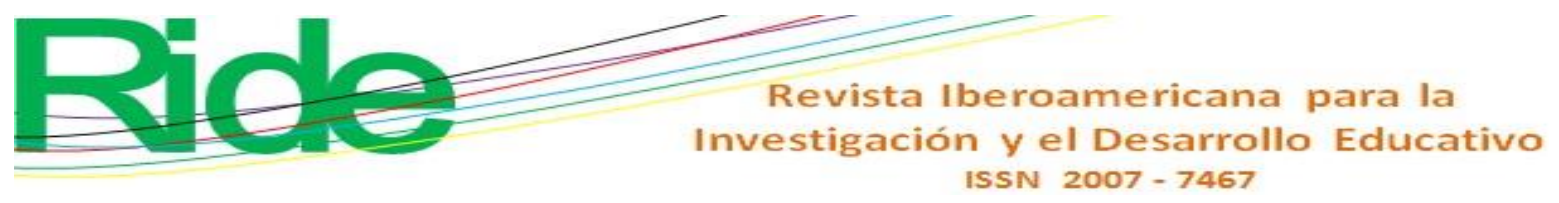

y recursos tecnológicos que procuran potenciar el proceso enseñanza-aprendizaje. En conclusión, se puede indicar que los docentes emplean una pedagogía innovadora en los procesos formativos de los estudiantes, la cual se apoya en problemas reales de contexto y en distintas herramientas tecnológicas. Palabras claves: competencias del docente, estrategias educativas, innovación pedagógica, modelo educativo, recursos educacionales.

\section{Abstract}

The objective of the present article is to propose a model of educational innovation based on the experiences of teachers who were qualified as the best by their students and based on grounded theory. The realized study corresponds to a qualitative approach. The research involved 17 teachers and 25 students from a public University at the State of Sonora, Mexico. The acquired results expose the construction process of a didactic constructivist model with a social-formative approach on instructional strategies and formative evaluation, as well in the functions and humanist teaching roles based in respect, empathy, trust and with the use of media and technological resources to improve the teaching-learning process. It concludes that the teachers employ an innovative pedagogy in the formative processes of the students based on real context problematics and supported by technological tools.

Keywords: teacher qualifications, educational strategies, teaching method innovations, educational models and educational resources.

\section{Resumo}

O objetivo do presente estudo foi identificar as práticas inovadoras que os professores utilizam para desenvolver o processo de ensino-aprendizagem em sala de aula, a fim de desenvolver uma teoria fundamentada sobre inovação educacional existente na prática de ensino. A pesquisa envolveu 17 professores e 25 estudantes de uma universidade pública no estado de Sonora, no México. Os resultados obtidos revelam um modelo didático construtivista com abordagem socioformativa nas estratégias de avaliação didática e formativa, bem como papéis de ensino humanista e baseados no respeito, empatia e confiança, apoiados no uso de meios e recursos tecnologia que busca aprimorar o processo de ensino-aprendizagem. Concluindo, pode-se indicar que os professores utilizam uma pedagogia inovadora nos processos formativos dos alunos, baseada em problemas reais de contexto e em diferentes ferramentas tecnológicas. 


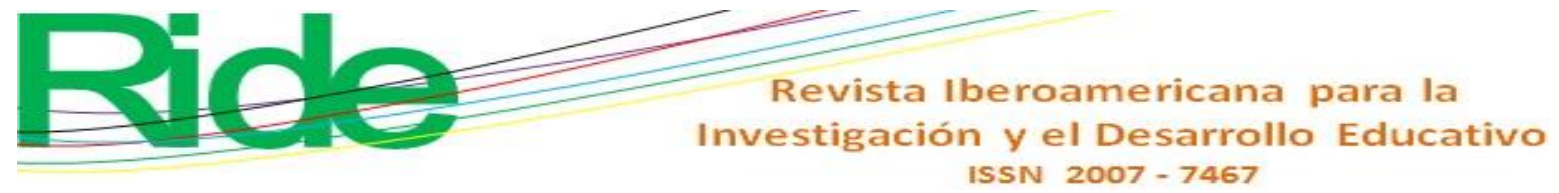

Palavras-chave: competências dos professores, estratégias educacionais, inovação pedagógica, modelo educacional, recursos educacionais.

Fecha Recepción: Julio 2019

Fecha Aceptación: Diciembre 2019

\section{Introducción}

El objetivo del presente estudio fue identificar las prácticas innovadoras que utilizan los docentes para desarrollar el proceso de enseñanza-aprendizaje en el aula con el fin de desarrollar una teoría fundamentada sobre la innovación educativa que existe en la práctica docente. La Organización de las Naciones Unidas (ONU) (2016) declaró que la innovación constituye un cambio que incide en algún aspecto estructural de la educación para mejorar su calidad, lo cual puede ocurrir a nivel de aula, de institución educativa y de sistema escolar. Del mismo modo, la Organización de las Naciones Unidas para la Educación, la Ciencia y la Cultura (Unesco) (2015) manifiesta que "el conocimiento y la innovación son importantes motores del desarrollo, el buen aprendizaje y una educación de buena calidad son cada vez más determinantes en el bienestar de los individuos, en el progreso del país y en la calidad de futuro común de la humanidad" (p. 9).

Se han presentado diversas percepciones sobre la innovación educativa, la cual se define como un proceso, cambio y mejora educativa que procura optimizar el aprendizaje de los estudiantes y contribuir a la calidad educativa. Sin embargo, vale acotar que no cualquier cambio o modificación implica innovar, pues esta debe ser intencional, planificada y evaluada para conocer el impacto de dichas transformaciones en el aula, en los alumnos y en los propios procesos de aprendizaje. Entonces, el significado de la innovación educativa presenta diferentes y similares interpretaciones, aunque de manera general se puede decir que es considerada como "una estrategia para avanzar en el logro de los fines institucionales. Su trayectoria aún es joven y requiere ampliarse, consolidarse y penetrar en todos los ámbitos de la institución, logrando así desarrollar una nueva cultura" (Ortega et al., 2007, p. 145).

Para Rodríguez, González y Gámiz (2016) la innovación educativa es un proceso que tiene que responder a las necesidades presentes en el ámbito educativo, de modo que debe ser eficaz y eficiente, sostenible y con resultados transferibles fuera del contexto de donde surgen. La innovación educativa, por ende, implica trasformaciones en la práctica docente. Barraza (2013) especifica cuatro ámbitos de la innovación educativa: incluir nuevos temas o contenidos al currículo, emplear nuevos materiales y 


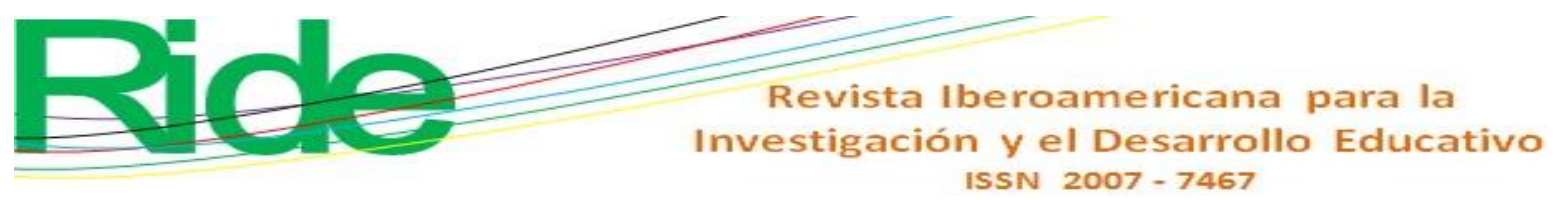

tecnologías, aplicar nuevos enfoques y estrategias de enseñanza aprendizaje, y el cambio de creencias y presupuestos pedagógicos de los diferentes actores educativos.

La innovación en el ámbito universitario representa considerar la calidad de la enseñanza universitaria y el desarrollo profesional del profesorado, las universidades han de desarrollar actividades de formación para contribuir al desarrollo profesional del profesorado universitario en ejercicio, actividades que puedan adoptar diversas modalidades formativas (Porto y Mosteiro, 2014, p. 145).

En consecuencia, la docencia universitaria requiere de cambios constantes y transformadores, para lo cual existe una alternativa: la innovación educativa:

Son los docentes los actores decisivos de los procesos de cambio educativo y las innovaciones, tanto pedagógicas como tecnológicas, solo son viables si cuentan con la complicidad y el protagonismo de los docentes. Asimismo, la innovación educativa demanda una cierta adaptación de las estructuras y los espacios escolares (Michavila, 2009, p. 4).

Según González (2014), la innovación en las aulas es un proceso inacabado de la reconstrucción de la docencia y de su fortalecimiento, de intercambio de estrategias didácticas transformadoras que respondan a los cambios en los procesos de enseñanza y a la promoción de los aprendizajes significativos.

\section{Educación innovadora}

Por lo antes expuesto, se han socializado innovaciones educativas entre docentes; por ejemplo, en dos universidades de España se realizó un estudio con enfoque cualitativo con el fin de examinar los contenidos de diversas convocatorias de proyectos de innovación para establecer un referente conceptual sobre la innovación educativa y sus significados teóricos.

A manera de hallazgos, según Rodríguez et al. (2016), se obtuvieron cuatro grandes ejes: “Los cambios en las metodologías de enseñanza, las tecnologías de la información y la comunicación, el bilingüismo y la generación, así como la difusión de buenas prácticas” (p. 194).

Asimismo, en Costa Rica se han implementado programas relacionados con la innovación pedagógica orientados a prácticas docentes consideradas exitosas. El propósito ha sido sugerir la implementación de procesos involucrando diferentes equipos que atiendan la docencia, la investigación, la elaboración y la práctica profesional de las diferentes carreras. De este modo, la 


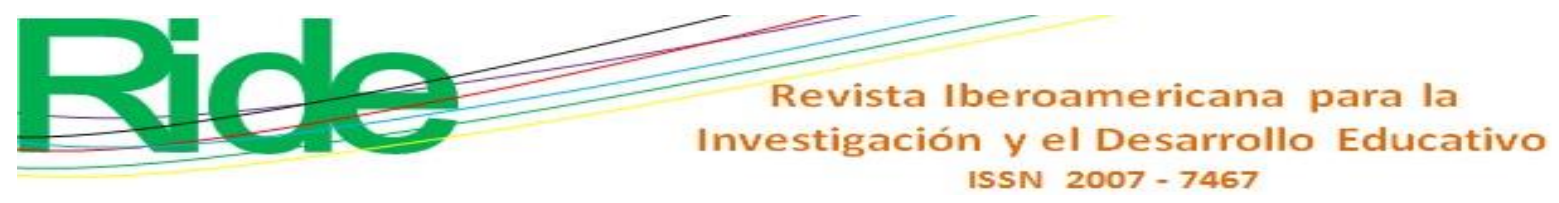

innovación pedagógica se visualiza — de acuerdo con Miranda, Angulo y Román (2018) — como "la introducción de un cambio en un determinado contexto o situación y que se da a través de estrategias a corto plazo con el fin de producir beneficios a través de acciones o actividades intencionales” (p. 6).

Asimismo, en Chile se han implementado metodologías activas centradas en el estudiante como desarrollo de innovaciones pedagógicas, lo que ha servido para demostrar un mejor desempeño en contenidos disciplinares de estudiantes de formación inicial, así como una percepción positiva de ellos en cuanto a las dinámicas de trabajo y a la interacción en el aula debido al papel de motivador que asume el docente (Maldonado y Rodríguez, 2016).

Otra metodología activa utilizada en las prácticas docentes ha sido el aula invertida [T-FliC], la cual se basa en el uso de recursos tecnológicos gratuitos (Google, Yotuube, entre otros) que se pueden implementar en distintas asignaturas en el nivel superior. Basso, Bravo, Castro y Moraga (2018) han comprobado que esta propuesta tecnológica propicia la interacciones de comunidades docentes que pueden compartir sus experiencias y ampliar espacios físicos hacia uno virtual que favorece la comunicación en distintas comunidades docentes con alcance nacional e internacional. Además, para el estudiante brinda un ambiente de aprendizaje flexible, así como una retroalimentación permanente y oportuna, y una evaluación formativa con énfasis en la reflexión.

Guzmán, Vargas, García, Núñez y Olmos (2016) también llevan a cabo una investigación para diseñar y poner en práctica un modelo de innovación educativa que permita identificar las áreas de oportunidad y las fortalezas de los profesores. De este modo, se procura generar un plan de trabajo y seguimiento personalizado a los docentes de la institución. A partir de los resultados del diagnóstico de dicho estudio, se determinó que los docentes utilizan herramientas tecnológicas como Nearpod (herramienta web/app que permite crear presentaciones interactivas), Google Form (aplicación de Google Drive para realizar formularios y encuestas), Wolfram Alpha (buscador de respuestas) y simuladores. Asimismo, participan en proyectos de Novus (proyectos institucionales que buscan resolver un problema de aprendizaje) y asesoran a otros profesores. Incluso han sido evaluadores de proyectos de innovación educativa en el congreso de innovación y utilizan las técnicas didácticas como Flipping the classroom (modelo pedagógico que transfiere el aprendizaje fuera del aula), POL (técnica para encontrar soluciones a trabajos en diversos escenarios) y método de caso. Para evaluar aplican examen presencial, quiz presencial y virtual, así como exámenes en equipo. Aunado a esto, emplean plataformas digitales para suministrar contenidos, administrar el curso y evaluarlo (Guzmán et al., 2016). 


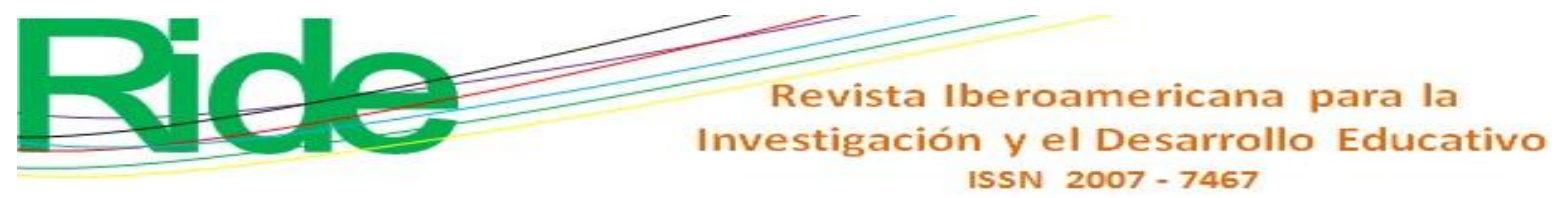

Por otro lado, existen diversas investigaciones sobre métodos de enseñanza con un enfoque socioformativo, como el caso de Tobón (2014), quien menciona que "la socioformación busca formar personas con un sólido proyecto ético de vida, emprendimiento, trabajo colaborativo, creación del conocimiento y metacognición para resolver problemas en contextos cambiantes y complejos” (p. 40). En este sentido, Parra, Tobón y López (2015) explican que “desde la socioformación se propone el desarrollo de competencias sustentables en los diversos actores sociales (estudiantes, docentes, directivos, políticos, líderes, entre otros), las cuales se refiere a la solución de problemas en situaciones complejas y cambiantes" (p. 43). Los autores se enfocan en generar nuevas alternativas que brinden medidas adecuadas para solucionar problemas en situaciones reales, mediante proyectos de innovación e investigación que exigen saber conocer, saber hacer, saber convivir y saber ser. En concordancia con esta idea, Gerlach y Velásquez (2010) afirman que "la socioformación está adquiriendo una gran importancia, por lo que cada vez existe mayor preocupación por las tareas de la formación. Educar implica tanto instruir como formar personas con determinadas habilidades y actitudes" (p. 40).

A partir de un estudio en la Universidad Autónoma de Chihuahua, que tuvo como meta determinar la relación entre la docencia socioformativa y el desempeño académico en los estudiantes universitarios, se evaluaron diversas variables relacionadas con la docencia desde la socioformación. Encontraron que cuando los estudiantes muestran interés por aprender se suelen motivar por la solución de problemas reales y por ejecutar proyectos aplicados. Por ello, se determinó que la docencia socioformativa atiende intereses de los docentes y estudiantes, y contribuye a la formación integral del estudiante (Parra et al., 2015).

Según lo anterior, puede considerarse al enfoque socioformativo como una innovación para la institución educativa, pues se presenta como un cambio referente al papel que juega el maestro, el alumno y las competencias que se deben fomentar. Sin embargo, es importante comentar que se está manifestando una prueba piloto en una de las carreras de dicha institución educativa en México (Sonora), la cual debe ser examinada para precisar si los docentes están generando y empleando prácticas innovadoras $\mathrm{u}$ otros enfoques, teorías y modelos considerados transformadores de los procesos de enseñanza-aprendizaje.

Por tanto, una vez precisadas las prácticas educativas innovadoras, se puede proponer un modelo de innovación educativa sobre la base de las experiencias de los docentes mejor calificados por sus estudiantes, lo cual se sustenta en la teoría fundamentada. En tal sentido, surge la siguiente 


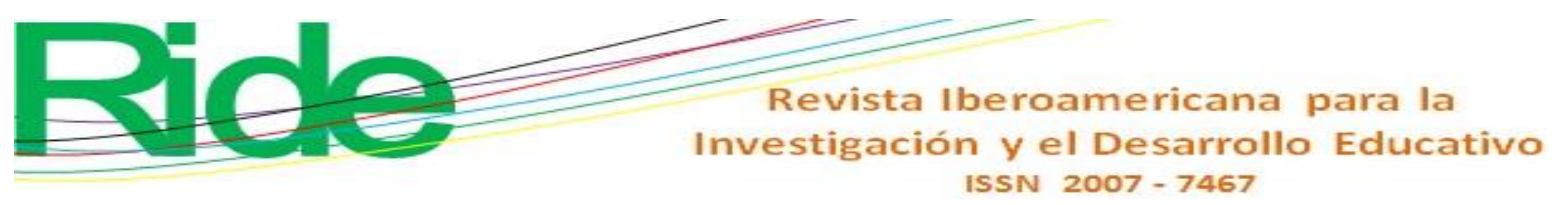

interrogante: ¿qué elementos aportan las prácticas docentes al modelo de innovación educativa según la percepción de estudiantes y docentes?

\section{Materiales y método}

El enfoque del presente estudio fue cualitativo debido a que se trata de una investigación que recaba información de acuerdo con la percepción de los docentes y estudiantes en torno a la temática de innovación educativa en el aula. El diseño de la investigación corresponde a una teoría fundamentada, la cual — según Vivar, Arantzamendi, López-Dicastillo y Gordo (2010) — se sustenta en la teoría del interaccionismo simbólico con el fin de comprender cómo definen los individuos un fenómeno o acontecimiento a partir de su interacción social. El propósito, en definitiva, es estudiar los fenómenos sociales en contextos naturales para generar teorías que puedan explicarlos.

La técnica usada para recabar los datos consistió en un grupo focal, el cual es definido por Hamui y Varela (2013) como "un espacio de opinión para captar el sentir, pensar y vivir de los individuos, provocando autoexplicaciones para obtener datos cualitativos" (p. 56). Esta técnica se basa en una entrevista informal grupal en la que normalmente participan entre 5 y 12 personas a quienes se les solicita que expresen sus opiniones, actitudes, creencias, satisfacción y percepciones sobre el tema propuesto.

Para elegir a los docentes se consideró un muestreo no probabilístico. En síntesis, se seleccionó solo a aquellos docentes con resultados sobresalientes en las últimas tres evaluaciones que los alumnos les realizaron sobre sus desempeños en clases. La técnica de muestreo fue por conveniencia o voluntarios, ya que los sujetos fueron seleccionados dada su proximidad.

En total, participó un grupo de 17 docentes de una universidad ubicada en el sur de Sonora (México), de los cales 8 eran mujeres y 9 hombres. El rango de sus edades osciló entre los 32 y los 62 años. Sus profesiones o carreras eran las siguientes: licenciados en Administración (LA), licenciados en Contaduría Pública (LCP), licenciados en Ciencias de la Educación (LCE), licenciados en Informática (LI), licenciados en Administración de Empresas Turísticas (LAET) y licenciados en Psicología (LPS), así como ingeniería (Ing.), ingeniería en Bioquímica (IB) y maestría en Administración (MA).

Con respecto al grado de estudios, 14 tenían el grado de maestría, 2 el grado de licenciatura y uno era pasante de posgrado. Las últimas universidades en las que estudiaron fueron las siguientes: Instituto Tecnológico de Sonora, Universidad de Sonora, Tec Milenio, Universidad de Durango, 


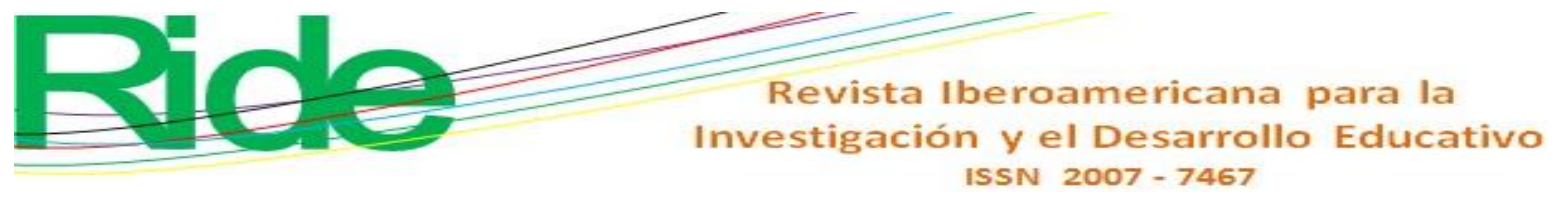

Instituto Tecnológico del Mar, UNO Internacional, Universidad Durango Santander, Instituto Pedagógico de Posgrado en Sonora y Universidad de Guadalajara.

Los 17 docentes contaban con capacitación en el área de docencia; de hecho, 16 de ellos tenían capacitación en TIC, mientras que 10 tenían experiencia en investigación con participaciones en ponencias, capítulos de libros, revistas o libros. Asimismo, 14 habían participado en la reestructuración curricular (p. ej., en la elaboración de programas de curso o planeaciones) y 8 habían fungido como tutores.

En el caso de los estudiantes, se optó por un muestreo probabilístico de manera aleatoria, lo que constituye un método mediante el cual todos los alumnos cuentan con las mismas posibilidades de ser incluidos. En concreto, participaron 25 universitarios de distintas carreras, tales como LAET, LCE y LPS, todos de la misma institución y cursantes del sexto semestre con un rango de edades que oscilaba entre los 20 y los 22 años, seleccionados de forma aleatoria.

Para ambos grupos de participantes se elaboraron guías de tópicos, las cuales consisten en un documento que conduce las herramientas necesarias para realizar un estudio. En el caso de los maestros, se formularon 13 ítems referentes al tema Innovación educativa en el aula, los cuales fueron divididos en tres categorías y diversos enfoques, así como una conclusión. Para las preguntas que contenían la guía de tópicos se consideraron las categorías perspectivas de la innovación educativa y funciones de la docencia. La primera estuvo constituida por los indicadores tendencias educativas y enfoques curriculares. La categoría funciones de la docencia quedó conformada por los indicadores de didáctica (técnicas y métodos de enseñanza), interacción (tutoría), uso de las TIC y evaluación.

Para los estudiantes se diseñaron 10 reactivos divididos en cuatro categorías. La primera se refería a las características del docente innovador; la segunda abarcaba las estrategias o actividades en clase; la tercera se relacionaba con los recursos educativos, y la última se vinculaba con las formas de evaluación.

El procedimiento se enfocó en la elaboración del instrumento, el pilotaje, la aplicación del grupo focal y la recolección de los datos obtenidos. La elaboración del instrumento consistió en la realización del cuadro de operacionalización de las variables. Asimismo, se elaboró un análisis con el fin de identificar las dimensiones usadas para desarrollar categorías y mediciones con base en la información recabada, cuya finalidad se centró en el diseño de la guía de tópicos implementada en el grupo focal.

Antes de aplicar la prueba piloto, el instrumento fue validado por tres maestras de educación del área de investigación educativa expertas en el tema, las cuales aportaron algunas recomendaciones sobre la redacción de las preguntas. De este modo se aseguró que las interrogantes correspondieran 


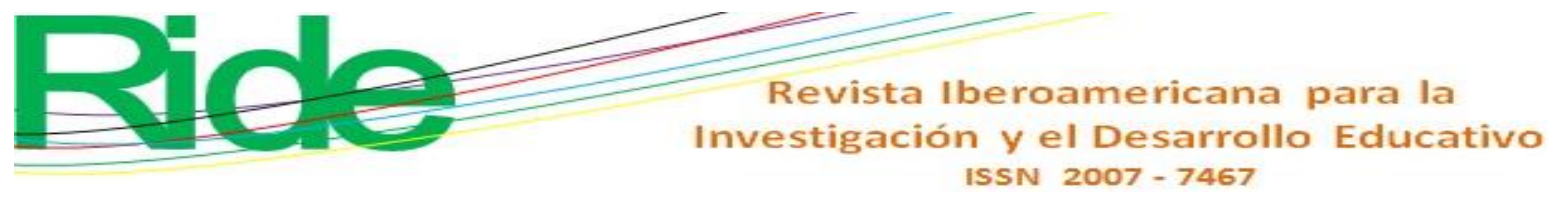

con la dimensión propuesta. Además, para la confiabilidad de las preguntas se trabajó con docentes y con estudiantes para corroborar que fueran claras, precisas y fidedignas.

La aplicación de la guía de tópicos se efectuó a partir de grupos focales con docentes y estudiantes previamente organizados por un comité organizador cuyas funciones eran fungir como moderador o facilitador, secretario y asistente técnico.

El moderador o facilitador es aquel que conduce la sesión y enfoca la discusión por medio de preguntas abiertas; el secretario recaba información de manera escrita, mientras que el asistente técnico se encarga de grabar los comentarios, observar el ambiente y apoyar al moderador y al secretario.

En lo que concierne a la ejecución del grupo focal Prácticas de innovación educativa en el aula, se obtuvieron tres mesas focales con un total de 7 docentes en cada una, los cuales impartían clases para diferentes carreras. En consecuencia, en cada área se recabó información de manera distinta debido a que se encontraban docentes de diferentes profesiones, los cuales generaban una diversidad en las respuestas, pues los encuestados o participantes implicados en la sesión del grupo focal comentaron, argumentaron y debatieron sus opiniones referentes al tema central y desde su programa educativo. En cuanto a los estudiantes, estos fueron distribuidos en tres mesas con siete sujetos de las diferentes carreras antes mencionadas.

El grupo focal tuvo una participación promedio de una hora con veinte minutos; durante la sesión se captaron las respuestas por medio de grabadoras digitales y se trascribió la información en el procesador de texto de Word. El análisis correspondiente se realizó por medio de tablas de saturación según los segmentos de los testimonios de los participantes.

Con base en la revisión teórica, emergieron las categorías de análisis y los códigos, validados por el equipo experto en innovación educativa. Recabada la información más relevante acerca de los testimonios de los participantes, se sometieron los análisis correspondientes a las tablas de saturación en conjunto con los expertos sobre el tema de innovación y uso de la tecnología en la educación. Para confiabilizar el proceso se discutió la codificación abierta con la codificación teórica entre tres expertos para acordar la categoría asignada.

Luego de esto, se utilizó el software MAXQDA Analytics Pro 2018 para que cumpliera con la tarea de procesar información mediante códigos sustantivos, es decir, datos empíricos. De esta forma, se desarrollaron códigos teóricos que se iban registrando a partir de los memos que consisten en ideas teóricas sobre los códigos y sus relaciones, tal como le surgen al analista mientras se codifica. Del mismo modo, se identificaron los códigos vivos que procedían directamente del lenguaje empleado 


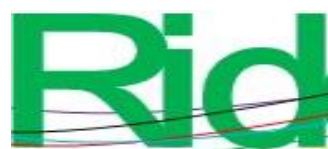

Revista Iberoamericana para la Investigación y el Desarrollo Educativo

ISSN $2007-7467$

por los informantes, como respaldo al sistema de categorías que emergieron del análisis y con el fin de obtener una teoría fundamentada.

\section{Resultados y discusión}

El proceso de codificación para el desarrollo de categorías y subcategorías correspondió al marco propuesto por Strauss y Corbin (2002), a partir de la codificación abierta donde se identificaron los conceptos y dimensiones que emergieron del análisis de los informantes (estudiantes y maestros). En este sentido, se asoció cada concepto con un color y así para cada categoría y subcategoría, asignando un código apoyado con los memorandos en relación con la teoría. En la figura 1 se identifican las categorías que emergieron.

Al ubicar las categorías y subcategorías relacionadas se explican los resultados según lo propuesto por Strauss y Corbin (2002) con la codificación axial y posteriormente la codificación selectiva, la cual consiste en seleccionar la categoría central en torno al análisis de las otras categorías, como se muestra en la figura 1. 
Figura 1. Análisis de datos cualitativos con el software MAXQDA

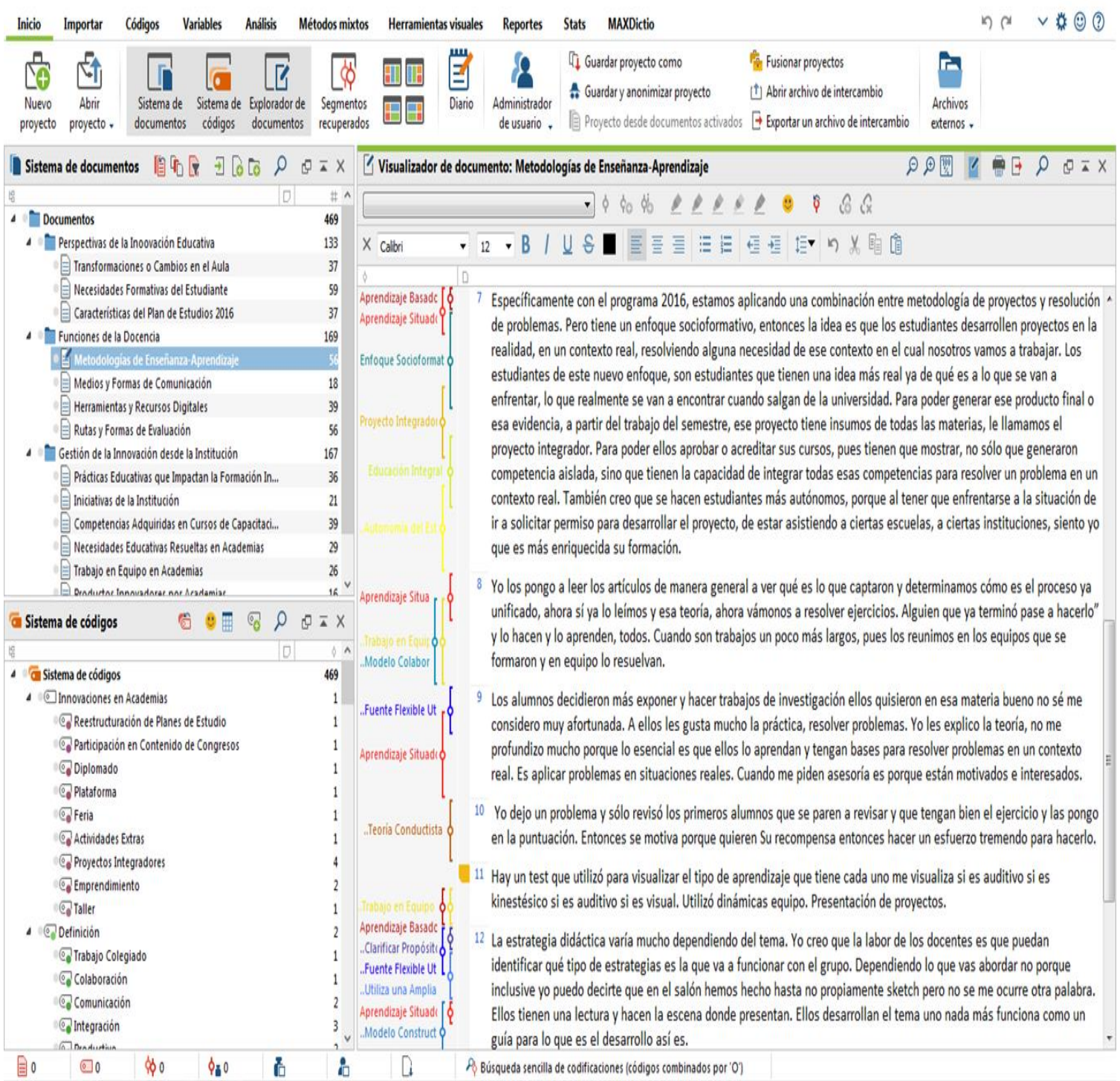

Fuente: Elaboración propia

Ante la pregunta ¿qué prácticas docentes innovadoras emplean los maestros para desarrollar el proceso de enseñanza aprendizaje en el aula?, las categorías obtenidas de las unidades de estudio fueron las siguientes: de acuerdo con las respuestas de los docentes, la primera se identifica con el modelo didáctico constructivista, que consiste en desarrollar capacidades. En este "se fomenta mucho lo que es el trabajo en equipo, hacer que el estudiante sea más autónomo, que el estudiante adquiera el aprendizaje a través de sus necesidades de formación” (D5). Asimismo, los docentes señalan lo siguiente: "Me funciona mucho que ellos construyan el conocimiento a base de ideas que ellos generan en el grupo, el propio alumno pueda construir el conocimiento dentro del aula y pueda gestionar su crecimiento" (D2). 


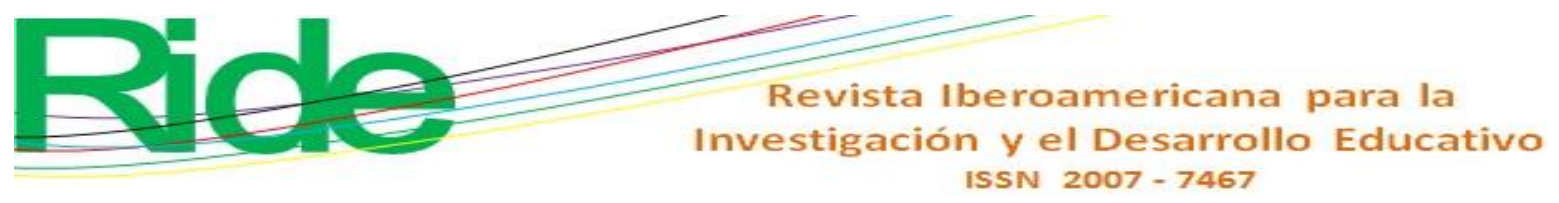

De igual forma, los profesores identificaron como categorías axiales al modelo constructivista con la teoría humanista, la cual se destaca entre las demás teorías, pues obtuvo 13 menciones. Esta promueve el desarrollo integral y ayuda al educando a convertirse en persona, reconociendo su libertad. Asimismo, genera la enseñanza de valores y actitudes, promueve la empatía y brinda confianza para obtener un impacto favorable en el aprendizaje significativo mediante la práctica. Se trata de "ser más empático con el alumno" (D11), a través de "comunicación de respeto, ser amigos de los alumnos, que entren en confianza, siempre apoyarlos y nunca intimidarlos, amenazarlos, ni hacerles sentir miedo; por eso, debe haber esa amistad, pero no de fiesta, sino de respeto y de que se sienta el apoyo al alumno" (D4).

Dentro de esta teoría destacan como características los siguientes valores: respeto, empatía y confianza, por lo que resulta sobresaliente para la institución el que los docentes sustenten sus prácticas en la teoría humanista, ya que esta brinda un énfasis en los educandos y su desarrollo como personas.

En cuanto a los estudiantes, mencionaron cuatro técnicas de enseñanza que emplean sus maestros, entre los cuales se destaca el aprendizaje colaborativo teórico-práctico, que tuvo mayor frecuencia (60\% de las opiniones). Por ejemplo, se expresaron sobre "las prácticas profesionales, pues uno va a todo lo que ha aprendido y lo pone en práctica en una escuela" (E3); otra opinión señala "las prácticas, como dicen mis compañeros, ya que pues nosotros practicamos y realizamos las cosas y de esa manera nosotros adquirimos las cosas, las habilidades esas necesarias para la carrera" (E6).

Aunado a lo anterior, el aprendizaje colaborativo también se presenta con siete repeticiones, lo que equivale a $18 \%$ de las opiniones de los estudiantes. Una de ellas es la siguiente: "Las estrategias que pudieran utilizar los maestros serían por ejemplo el trabajo en equipo, que de esa manera los alumnos aprenden un poco más, pero entre todos apoyándose” (E7).

También se obtuvieron percepciones sobre los modelos didácticos que implementan los docentes en el proceso de enseñanza-aprendizaje; en dicha cuestión, el modelo constructivista fue el más referenciado con $75 \%$ de las opiniones: "Pues que nos quede un mejor aprendizaje y que lo podamos poner en práctica” (E5). "Cuando estemos en la práctica o cuando estemos en el ámbito laboral de cómo mejorar nosotros para poder dar las clases" (E4). Según lo anterior, las cifras y los comentarios de los estudiantes, se puede afirmar que se trabaja predominantemente con los postulados del modelo constructivista. 


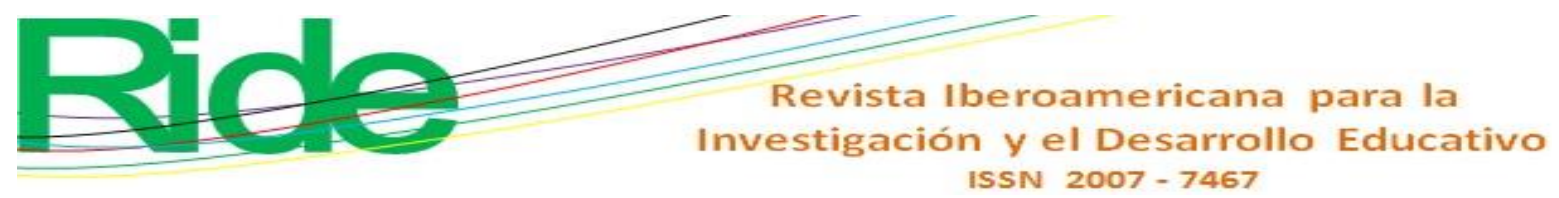

Por otro lado, los estudiantes identificaron el rol docente. Al respecto, los alumnos opinaron lo siguiente: “Considero que los docentes deben de ser más dinámicos, estar abiertos a diferentes formas (...), implementar nuevas formas, así los estudiantes puedan aprender más” (E7). Otro participante mencionó: "Bueno, yo creo que una característica puede ser que sí, que el maestro sea de mente abierta, que escuche la opinión de todos o que sea abierta en el sentido de que (...) ahora voy a intentar esto en el sentido de que voy a ser mejor, puede ser que el alumno aprenda mejor" (E1).

Por otra parte, la segunda codificación axial identificada fue el enfoque socioformativo. Este se relaciona con el modelo constructivista, ya que permite al alumno crear el conocimiento a través de la acción, pues se toma en cuenta el aprendizaje de competencias necesarias para la autorrealización, dado que apoya el acercamiento y entrelazamiento de las instituciones educativas con la sociedad. Al respecto, los docentes opinaron lo siguiente: "La idea es que los estudiantes desarrollen proyectos en un contexto real, resolviendo alguna necesidad; los estudiantes de este nuevo enfoque tienen una idea más real de qué es a lo que se van a enfrentar, lo que realmente se van a encontrar cuando salgan de la universidad" (D14). Además, resaltaron como característica el enfrentarse a la realidad: "Estamos formando a estudiantes para que sean competentes en cualquier ámbito de trabajo, de sus respectivos giros y en cualquier parte de la república, hacer los análisis correspondientes para que lleguen a resultados concretos y se les pueda retroalimentar" (D7). Es decir, resolver problemas en diferentes contextos: "Permitir al alumno egresar y ser suficientemente competente en el ámbito laboral" (D4), destacando el desarrollo de competencias.

Los estudiantes, por su parte, comparten opiniones de alcanzar un mayor aprendizaje cuando los docentes desarrollan estrategias de ejecución o apoyo. Esta categoría obtuvo siete puntos de frecuencia y $73.5 \%$ en las opiniones. Algunas de las percepciones más relevantes fueron: "Por ejemplo, que puede expresarme más por ejemplo si es un capítulo nuevo, que me toque un tema, o sea, que él me asesore, o que esté ahí para ayudarnos, o sea, me ayuda a comprenderlo más o, cómo le digo, que esté siempre ahí, pues ya, en el salón o fuera de él que nos dé asesorías” (E6). Por otro lado, las estrategias de incorporación o metacognitivas equivalen a $20.6 \%$, mientras que las de procesamiento o cognitivas obtuvieron solo dos puntos, equivalentes a $5.9 \%$. Una de las opiniones mencionada fue esta: "Adquirir mejor conocimiento, tener más claras las clases que nos implementan" (E3).

De la categoría central surge la clasificación teórica que según los memos generados en la codificación abierta y teórica se identifica con la dimensión competencias docentes, que cuenta con dos subdimensiones: funciones y roles docentes. Estas competencias son consideradas como el 


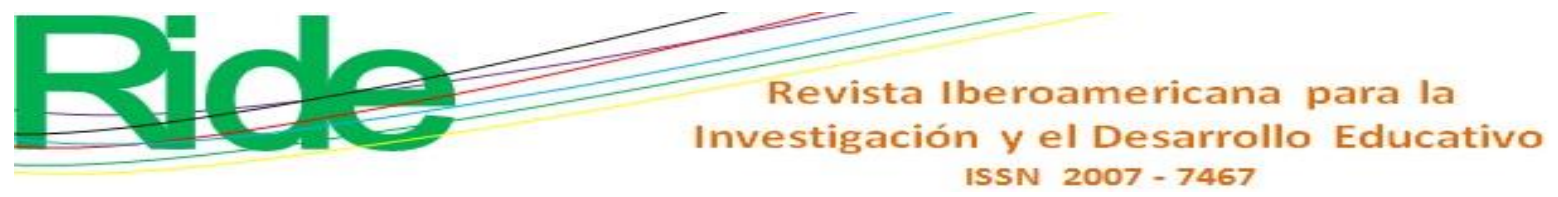

conjunto de decisiones de los docentes para desarrollar procesos formativos de calidad y promover el desarrollo de los estudiantes. Por ello, es esencial trabajar en la interacción didáctica, la metodología, la elaboración de medios y tareas (ambientes de aprendizaje), la acción tutorial y la evaluación (Domínguez, Medina y Cacheiro, 2010).

En cuanto a la subdimensión roles docentes, esta corresponde a los papeles que los profesores de la institución desempeñan, los cuales se empelan para ser categorizados como innovadores. Según Carcelén (2004), un docente innovador "se esfuerza por organizar y hacer disponibles la más amplia gama de recursos para el aprendizaje (p. 7). Este rol fue mencionado en ocho ocasiones por los docentes, como se muestra en seguida: "Dependiendo de lo que vas abordar, porque inclusive yo puedo decirte que en el salón hemos hecho sketch" (D10).

Continuando con esta idea, Carcelén (2004) considera que el docente tiene mucho que ver con establecer la disposición de ánimo general o el ambiente de la experiencia del grupo o de la clase. Esta variable fue mencionada siete veces por los docentes: "Crear un clima de aprendizaje favorable entre el grupo" (D3); "Clases más interactivas, no solamente utilizando la tecnología, sino también haciendo movimientos de lugar en donde se está impartiendo la clase; [así] existe mucha más participación y fluyen mucho mejor las ideas" (D6), lo que genera un ambiente de aceptación en la sala de clases.

En lo que concierne a la subdimensión funciones, se identificó solamente a la acción tutorial como una labor que desempeña el docente. Medina (2015) la define como el conjunto de decisiones que estimulan a cada estudiante, equipos y clases para que construyan su línea de desarrollo personal y profesional, aprovechando lo más valioso de los procesos instructivos y de los proyectos personales y profesionales.

De acuerdo con uno de los docentes consultados, se trata de "hacer un acercamiento, conocerlos un poco e identificar sus debilidades, de esa forma apoyarlos a que se desarrollen dentro de lo académico" (D7). Esto para realizar el proceso de enseñanza-aprendizaje en confianza mutua y flexible, donde el docente pueda asesorar al estudiante para cumplir con sus metas establecidas.

Es importante mencionar que la acción tutorial que llevan a cabo los docentes se relaciona directamente con la dimensión teoría humanista, ya que las características de respeto, empatía y confianza son necesarias para que los alumnos se sientan en un ambiente de confianza que les permita realizar ese acercamiento para expresar sus necesidades educativas o problemas académicos.

En cuanto a los estudiantes, se obtuvieron cinco categorías similares en el menor rango de frecuencia con $3.2 \%$, tales como las características de las buenas prácticas/cambios que implican mejoras en la calidad; de igual manera, la función docente en cuanto a la metodología de enseñanza 


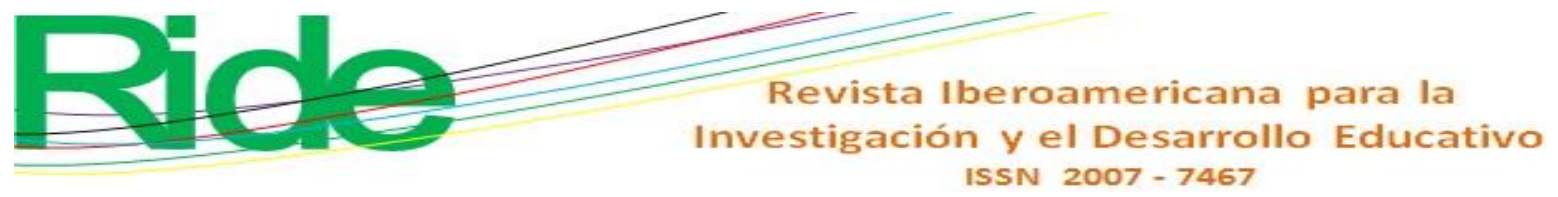

$\mathrm{y}$, aunque con menor frecuencia, el rol docente de acuerdo con la toma de iniciativas para compartir con el grupo, el ser capaz de transformarse en un estudiante participante y aceptar tanto el contenido intelectual como las actitudes emocionales. Por otra parte, la categoría con mayor frecuencia se encuentra relacionada con la función docente, en cuanto a la metodología de enseñanza con nueve repeticiones por parte de los participantes, semejante a $23.1 \%$ con respuestas como las siguientes: "Emplea nuevas técnicas. Utiliza nuevas técnicas para que el estudiante comprenda un tema nuevo, propone utilizar diferentes métodos de aprendizaje hacia los alumnos" (E1).

Estas opiniones tienen el mismo significado que el planteado por Medina y Salvador (2009) sobre las técnicas de enseñanza:

Los profesores principiantes necesitan sin duda un amplio repertorio de estrategias y técnicas de enseñanza que funcionen con los estudiantes a los que enseñan y en el contexto en que trabajan; necesitan estrategias para planificar, desarrollar y evaluar el currículum aplicado a un contexto diverso, un espacio escolar con alumnos claramente diferentes (p. 5).

Sin embargo, la categoría con menor frecuencia fue acción tutorial con una mención, lo que corresponde a 2.6\%. Los estudiantes opinaron: "Que siempre está al día con las actividades y que esta pendiente de sus alumnos" (E2). La acción tutorial, al obtener solo una mención, indica que los docentes de educación superior están centrados solamente en lo que indica el programa o plan de estudios, a pesar de que el alumno necesita un guía o un formador que se preocupe por sus necesidades y que se preste para ayudar a cubrirlas. La única opinión sobre la categoría acción tutorial puede estar relacionada con lo sostenido por Gerlach y Velásquez (2010) quienes argumentan:

Resulta de suma importancia plantear la necesidad de la formación de docentes en su ámbito de actuación, en la medida que muchos tienen diferentes tipos de formación profesional, sin embargo, no basta con saber la asignatura o los contenidos para ser docentes (p. 84).

La dimensión estrategias de enseñanza-aprendizaje es una categoría axial que se relaciona con la metodología que los docentes utilizan en el desarrollo de sus clases, como lo es el aprendizaje situado, que resultó ser la más empleada entre los docentes con 13 menciones. De acuerdo con Medina (2015), permite construir su propio aprendizaje con elementos de su contexto, dado que es un enlace entre la teoría y la práctica. De manera similar, los docentes consideran que "se le da una breve explicación al alumno del tema que se va a tratar y después se le pone un caso práctico, donde el alumno pone su conocimiento, de ahí para desarrollar esa asignación” (D5). 


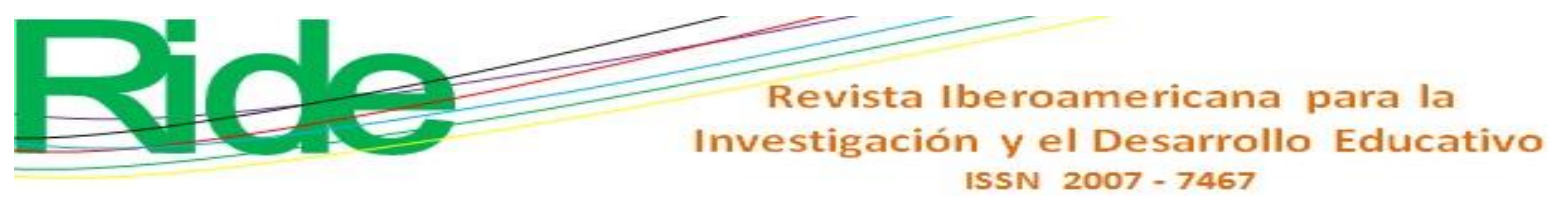

También se destacan el aprendizaje reflexivo y el basado en proyectos. El primero trata de una metodología que ayuda al estudiante a construir el conocimiento nuevo y sus experiencias previas mediante la interacción con otros individuos. Con nueve menciones, los docentes declararon que se trata de "dar más vivencias prácticas, desarrollar una teoría práctica, cualquier conocimiento que adquiera" (D11). Por su parte, el aprendizaje basado en proyectos, según Medina (2015), "es un esfuerzo que se lleva a cabo en un tiempo determinado para lograr el objetivo específico de crear un servicio o producto único, mediante la realización de una serie de tareas y el uso efectivo de recursos" (p. 44). Desde de la concepción de los docentes, "estamos utilizando el método de proyectos al final, pues el alumno elabora un proyecto y nos exponen, pues que ellos desarrollaron durante todo el curso" (D5). Este tipo de idea fue mencionada en siete ocasiones.

Asimismo, cabe mencionar que la dimensión estrategias de enseñanza-aprendizaje es una categoría axial que se relaciona directamente con otras dos dimensiones: evaluación del aprendizaje y medios y recursos tecnológicos, ya que al implementar las estrategias de enseñanza es necesario que los docentes evalúen los aprendizajes obtenidos por sus estudiantes. Igualmente, durante el desarrollo de las clases los maestros usan algunas tecnologías para complementar su metodología.

$\mathrm{Al}$ respecto, los consultados mencionaron cinco estrategias que utilizan los docentes, como las preguntas intercaladas (con 12 puntos de frecuencia equivalente a $43.3 \%$ ). Algunas opiniones fueron las siguientes: "Pues casi siempre hacemos preguntas: ¿Quién tiene alguna duda? Nos externan a que opinemos acerca de las dudas que nos quedaron acerca del tema anterior" (E1).

Las interrogantes intercaladas son señaladas en las estrategias de enseñanza. En el marco teórico de la investigación, Medina y Domínguez (2015) las definen como preguntas insertadas en la situación de enseñanza o en un texto. Estas tienen gran relevancia en el aprendizaje de los estudiantes, ya que se pueden entender como una actividad de dinamismo y motivación en un contexto semiiformal. Asimismo, se pueden interpretar como un mecanismo de retroalimentación que permite resolver dudas y expresar opiniones desde distintos puntos de vista.

Por otra parte, la categoría ilustraciones obtuvo poca frecuencia con $30 \%$. De hecho, en la presente interrogante se presenta una similitud en dos categorías (mapas conceptuales y redes semánticas), así como pistas, tipografías y discursivas, con solo una mención correspondiente a 6.7 \% .

La dimensión evaluación del aprendizaje, ubicada en las fases iniciales de la recolección de información, amerita el empleo de técnicas e instrumentos para tal fin (Arias y De Arias, 2011). En tal sentido, y para fortalecer este proceso, se tiene la subdimensión de estrategias, en la que se ubicó 


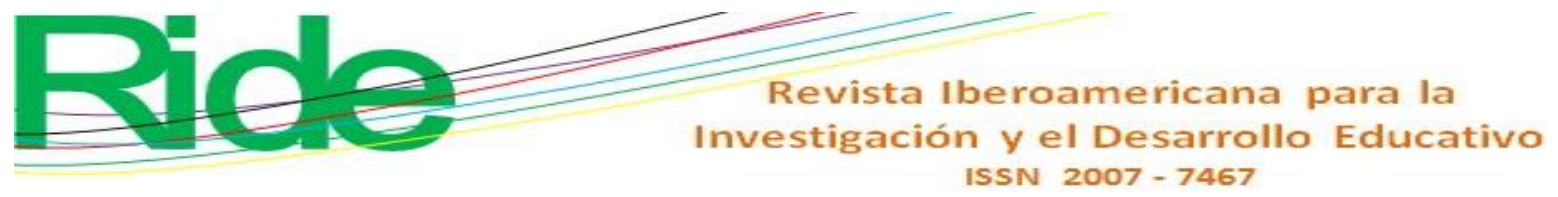

la evaluación formativa con ocho menciones. Uno de los comentarios fue el siguiente: "También la parte formativa ya sea en una exposición, examen escrito, en una exposición de un proyecto" (D1).

Además, se puede mencionar la evaluación sumativa con cinco menciones, la cual se define como aquella que "valora los resultados de aprendizaje y, por tanto, los procedimientos e instrumentos (...) [usados para] proporcionar información significativa acerca de lo que han aprendido las y los estudiantes (Secretaría de Educación Pública [SEP], 2013, p. 37). Sobre este elemento, los maestros mencionan: "La evaluación sumativa que se establece desde el principio, cuál es la ponderación que van a ir obteniendo por cada uno de los rubros y al final se adquiere una competencia y se le da una calificación" (D11).

No obstante, y de acuerdo con el modelo didáctico constructivista que se está implementando en la práctica docente, resulta vital centrarse en la evaluación diagnóstica, ya que este paradigma toma en cuenta los conocimientos previos para asociar el aprendizaje nuevo con el anterior.

En cuanto a la heteroevaluación, esta consiguió 11 menciones, la cual se refiere de forma general a la evaluación que realiza el docente del estudiante. Uno de los profesores opinó lo siguiente: "La heteroevaluación porque al final de cuentas la calificación definitiva la establece el docente como lo mencionaba el maestro de acuerdo a cuánto adquiriste y a qué tienes derecho" (D11).

La evaluación, lógicamente, consta de técnicas que permiten recolectar evidencias de aprendizaje de los estudiantes para ser analizadas e interpretadas para la toma de decisiones en relación con su aprendizaje. En este sentido, y como segunda subdimensión, se identificaron los instrumentos de evaluación, entre los cuales se destacan la rúbrica y el examen. La rúbrica permite identificar el nivel de desempeño que muestra el estudiante en el desarrollo de una actividad o problema (este obtuvo nueve menciones). Una consideración de los docentes fue esta: "La rúbrica la utilizo como una estrategia de retroalimentación porque ya la rúbrica nos dice si tuviste eso, pero te faltó todavía o te hace falta desarrollar tal cosa o estás excelente en tal área entonces lo que manejo" (D11). Con cinco menciones, los docentes explican: "Por ejemplo, tenemos un examen escrito después de haberlo hecho todos, se entregan los exámenes y se hace una retro de que de cuáles fueron los principales errores de los exámenes escritos" (D12).

En lo concerniente a los estudiantes, además de los exámenes, manifestaron técnicas de evaluación formal: la ejecución (con 21 puntos de frecuencia y 38.2 \% de las opiniones) pertenece a dicha categoría. Sobre este aspecto, uno de los alumnos consideró: "Podrían ser los proyectos finales, no te ponen examen, pero puedes hacer un proyecto, exposiciones" (E5). 


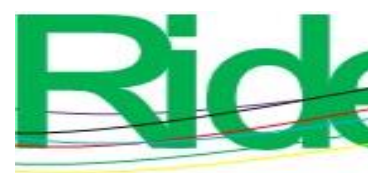

Revista Iberoamericana para la Investigación y el Desarrollo Educativo ISSN $2007-7467$

No obstante, la categoría semiiformal ejercicios y prácticas/tareas obtuvo $34.5 \%$ de las menciones, lo que la convierte en una de las categorías con mayor frecuencia. En cambio, la categoría menos frecuente fue observación, con solamente una mención, lo que equivale a 1.8 \%: "Pues también no, no simplemente evalúan por parte de exámenes, sino también evalúan tu actitud; ahora nos están evaluando mucho la actitud que tomemos frente a las cosas, o sea, a las actividades que ellos nos digan que tenemos que hacer; cuenta mucho la actitud que nosotros mostremos" (E1).

La dimensión Medios y recursos tecnológicos ayuda al docente a llevar a cabo el proceso de enseñanza, por lo que se tienen tres subdimensiones: didáctica, comunicación y uso de TIC. En primer lugar, la didáctica se refiere a la manera en que los docentes utilizan a la tecnología de forma innovadora para implementar sus sesiones. Esta se divide en los ambientes virtuales de aprendizaje y los medios de apoyo para la exposición oral.

En tal sentido, los entornos virtuales de aprendizaje juegan un rol muy importante en el ámbito de la educación, ya que no se trata solamente de tomar un curso y colocarlo en un ordenador, sino de combinar recursos, interactividad, apoyo y actividades estructuradas de aprendizaje (Limón, 2015).

Con un total de 14 menciones, los docentes consideraron que se trata de "dinámicas, tecnología y plataformas" (D13). Al mismo tiempo, mencionaron el "trabajar en la plataforma de Google Drive para compartir información" (D5), "las asignaturas, las tareas, las asignaciones las hago a través de la plataforma del Saeti, tiene que subir todas sus asignaciones, a mí no me entregan nada, todo lo dejan colocado ahí y yo les reviso a través de eso" (D9).

Por su parte, los medios de apoyo a la exposición oral son recursos cuya finalidad es combinar presentaciones tradicionales mediante una adecuada planificación para lograr una mayor eficacia como medio de aprendizaje. Con un total de ocho menciones, los docentes consideraron lo siguiente: "Primeramente los recursos que están inmediatos en el aula, contar con la computadora, el cañón, el pizarrón y ahora hasta pizarrón más chiquitos; esos recursos se utilizan, depende de la dinámica de que la clase esté preparada o tenemos para elegir; sí, a veces Power Point” (D8); “aprovechamos los recursos que tenemos en aula, en aula tenemos internet, tenemos proyector electrónico, nos apoyamos porque hoy en día tenemos una gran cantidad de medios visuales" (D9); "utilizamos en el aula que son videos presentación en Power Point y todo tipo de presentación que ellos utilizan” (D14).

En concordancia con la innovación educativa se encuentran la segunda subdimensión referente a los medios y recursos didácticos, es decir, comunicación, sobre la cual se tuvieron 12 menciones. Según Medina y Domínguez (2015), los nuevos recursos digitales posibilitan variadas estrategias para 


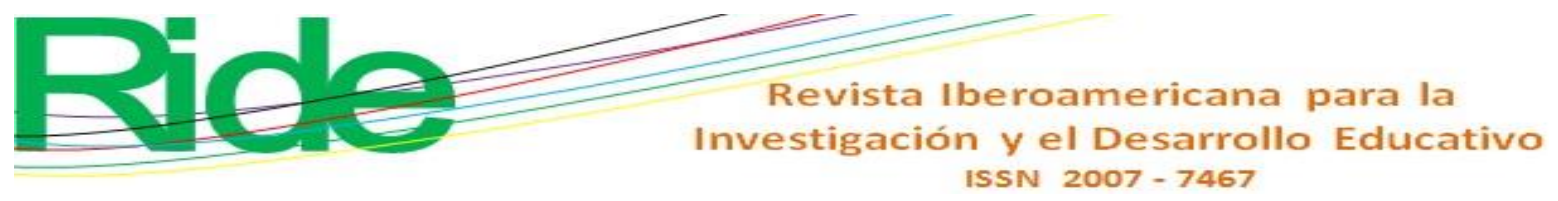

la interacción social, a través de e-books, documentos, foros, chats, correos, buscadores en internet, entre otros.

En tal sentido, y con un total de diez menciones, el correo electrónico resultó ser el principal medio de comunicación de los docentes consultados: "A mí en lo personal, lo que me ha funcionado más es el correo electrónico en mi celular" (D4). Además, algunos utilizan las redes sociales como Facebook y WhatsApp (con siete menciones cada una) para interactuar con sus estudiantes y resolver dudas: "WhatsApp y Facebook, más para comunicar algo urgente, alguna inquietud, alguna duda de algún alumno, entonces me ha funcionado" (D13); "ya les comparto mi teléfono, mi WhatsApp, y creo que los muchachos hacen más interacción conmigo para sacar dudas, preguntas, eso sí, yo tengo mis horarios; con correo de Gmail a mí también en mi celular, para mí es más rápido contestar correos" (D5).

Por otro lado, los estudiantes percibieron seis tecnologías, entre las cuales predominó la categoría WhatsApp/Facebook con una frecuencia de 16, equivalente a $36.2 \%$. Una opinión fue esta: "Para tener un contacto con los alumnos, muchas veces pues, crean un grupo de WhatsApp o Facebook y el maestro se comunica, sube las actividades, o en las exposiciones para que no se pierdan, en el WhatsApp pues muchas veces les avisan o a veces le pasan el número de teléfono y ya le mandan mensaje a un compañero, como al que ve más confianza y pues ese ya, para que le avise a todos si no va a poder asistir o si se cambió algo" (E3).

La opinión expuesta anteriormente se refiere al aprendizaje móvil, sin embargo, las plataformas también obtuvieron frecuencia significativa al contar con $25.5 \%$, sin gran diferencia con WhatsApp/Facebook, mientras que la menos mencionada fue foros con solo una mención, lo que corresponde a $4.3 \%$ de las opiniones. A continuación, en la figura 2, se expone el modelo de innovación educativa de la universidad: 
Figura 2. Modelo de innovación educativa en docentes de nivel superior

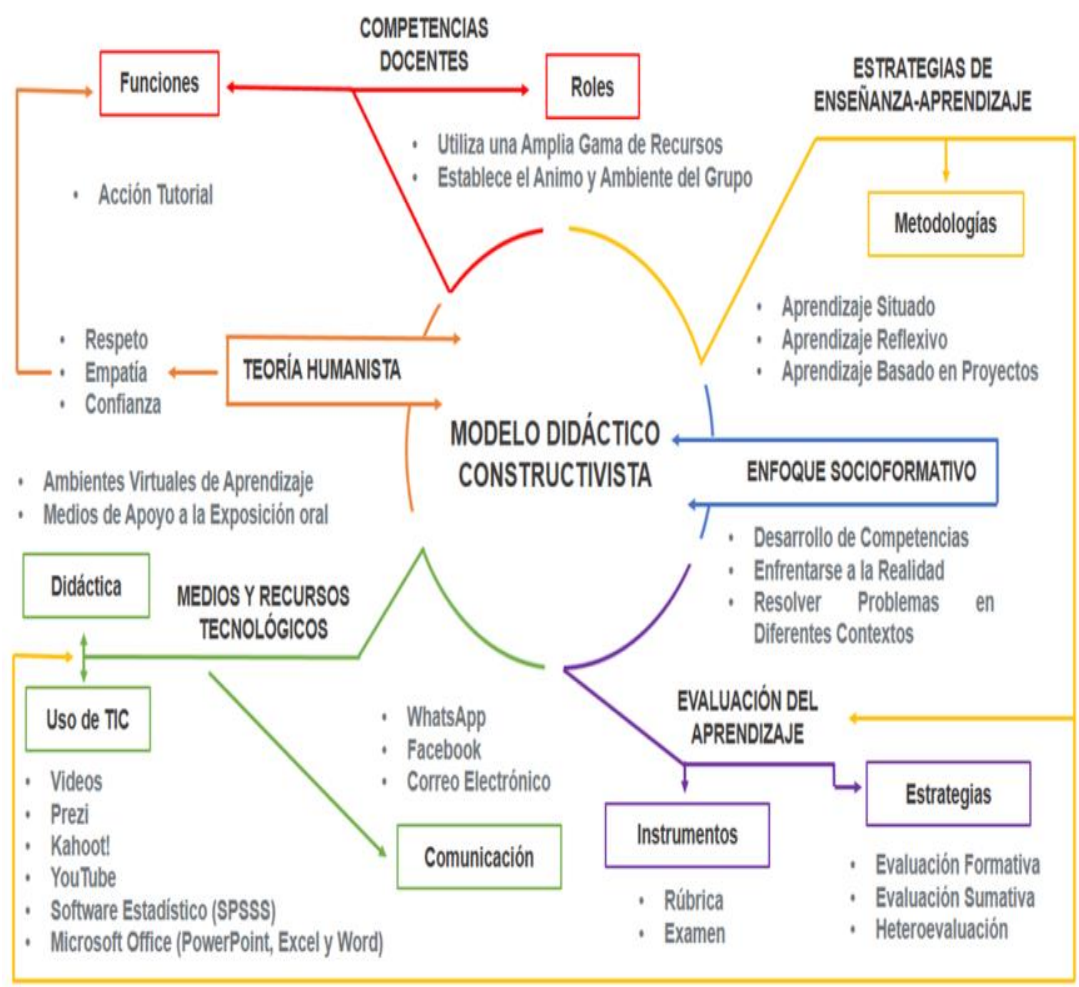

Fuente: Elaboración propia

\section{Conclusiones}

A partir de los resultados analizados, se puede concluir que el maestro de la universidad sonorense analizada trabaja predominantemente con el modelo didáctico constructivista (categoría central) y se basa en las codificaciones axiales de la teoría humanista y el enfoque socioformativo. En la teoría humanista se manejan los valores y actitudes de respeto, empatía y confianza, mientras que en el enfoque socioformativo el desarrollo de competencias, el enfrentarse a la realidad y el resolver problemas en diferentes contextos.

El modelo didáctico se conforma por cuatro dimensiones. La primera representa las competencias docentes, a partir de la Función de acción tutorial y los Roles del docente innovador, relacionados con una amplia gama de recursos para el aprendizaje, así como establecer el ánimo y ambiente de grupo. La segunda dimensión tiene que ver con las Estrategias de enseñanza-aprendizaje que los docentes implementan en sus aulas, basadas en las metodologías del aprendizaje situado, reflexivo y basado en problemas. 


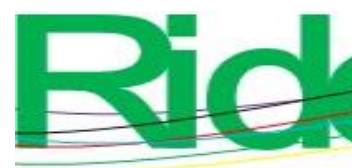

Revista Iberoamericana para la Investigación y el Desarrollo Educativo

ISSN $2007-7467$

La tercera dimensión, correspondiente a la Evaluación del aprendizaje, estuvo constituida por los momentos de la evaluación formativa, sumativa y la heteroevaluación como agente de evaluación, así como la rúbrica y el examen como principales Instrumentos.

La cuarta y última dimensión se refiere a los Medios y recursos tecnológicos que los maestros emplean para apoyar su enseñanza. En estos se halló la didáctica, equivalente a los ambientes virtuales de aprendizaje y al medio de apoyo para la exposición oral; asimismo, la comunicación se basó principalmente en el uso de correo electrónico y las redes sociales como Facebook y WhatsApp; además, las TIC que dichos docentes utilizan son los videos, Prezi, Kahoot!, YouTube, SPSS y Microsoft Office.

De acuerdo con los resultados recabados de forma general con los estudiantes y los docentes, se pueden destacar coincidencias en cuanto al rol del docente como flexible y mediador en los procesos de aprendizaje, empleo de estrategias didácticas reflexivas y basadas en problemas, y proyectos, estos últimos como estrategia de evaluación sumativa. Asimismo, hay congruencia al aplicar el aprendizaje situado, pues los estudiantes realizan prácticas profesionales que les permiten aplicar el conocimiento en un ambiente real. De hecho, concuerdan con utilizar redes sociales para establecer comunicación y el uso de plataformas para las tareas escolares.

Las diferencias en las percepciones se hallan en cuanto al rol del tutor, pues el docente cree que desempeña esta labor, mientras que los estudiantes expresan una baja frecuencia en este tipo de atenciones académicas. Asimismo, la evaluación diagnóstica no es identificada por ambos sujetos y la coevaluación es un agente que se deberá fortalecer desde la práctica docente socioformativa.

Finalmente, se debe resaltar que la innovación educativa es un proceso de cambio, de ideas propuestas y de aportaciones que sirven para mejorar la calidad de la educación por medio de conocimientos aplicados que deben situarse a través del tiempo para generar beneficios que impacten en la sociedad. Sin embargo, se debe tener en cuenta que la innovación no consiste en emplear el último software o dispositivo inventado, sino en aprovechar las tecnologías que se tienen al alcance para potenciar la práctica docente. 


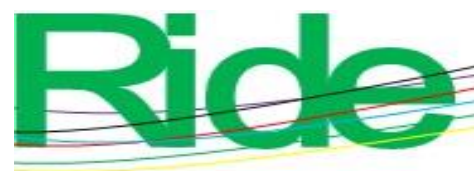

Revista Iberoamericana para la

Investigación y el Desarrollo Educativo

ISSN $2007-7467$

\section{Referencias}

Arias, S. y De Arias, M. (2011). Evaluar los aprendizajes: un enfoque innovador. Educere, 15(51), 357-368. Recuperado de http://www.redalyc.org/pdf/356/35621559006.pdf.

Barraza, A. (2013). ¿Cómo elaborar proyectos de innovación educativa? México: Universidad Pedagógica de Durango. Recuperado de https://redie.mx/librosyrevistas/libros/como_elaborar_proyectos_de_innovacion.pdf.

Basso, M., Bravo, M., Castro, A. y Moraga, C. (2018). Propuesta de modelo tecnológico para Flipped Classroom (T-FliC) en educación superior. Revista Educare, 22(2) 1-17. Doi: http://dx.org/10.15359/ree.22-2.2

Carcelén, C. (2004). Fundamentos teóricos para la innovación educativa. Recuperado de https://www.academia.edu/4071081/Fundamentos_te\%C3\%B3ricos_para_la_Innovaci\%C3 $\%$ B3n_Educativa.

Domínguez, M., Medina, A. y Cacheiro, M. (coords.) (2010). Investigación e innovación de la docencia en el EESS. Madrid: Ramón Areces.

Gerlach, L. y Velásquez, R. (2010). Formación docente en instituciones de educación superior de acuerdo al nuevo modelo curricular. En Angulo, J., Valdés, A., Mortis, S. y García, R. (eds.), Educación, tecnología e innovación (p. 40). Obregón, Sonora: ITSON.

González, C. (2014). Estrategias para trabajar la creatividad en la educación superior: pensamiento de diseño, aprendizaje basado en juegos y en proyectos. RED. Revista de Educación a Distancia, (40), 7-22. Recuperado de http://www.redalyc.org/articulo.oa?id=54730460002

Guzmán, M., Vargas, L., García, I., Núñez, M. y Olmos, N. (2016). Modelo de innovación educativa para desarrollo docente. Trabajo presentado en III Congreso Internacional de Innovación Educativa. Monterrey, México.

Hamui, A. y Varela, M. (2013). La técnica de grupos focales. Investigación en Educación Médica, 2(5), 55-60. Recuperado de http://www.redalyc.org/pdf/3497/349733230009.pdf.

Limón, R. (2015). La inclusión de recursos tecnológicos innovadores para enriquecer y potenciar el desempeño humano en la sociedad. En Gonzáles, I., Rodríguez, A., Zavala, M. y Vázquez, M. 


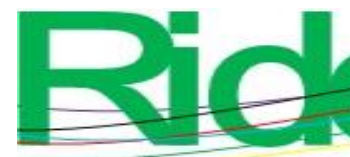

\section{Revista Iberoamericana para la Investigación y el Desarrollo Educativo ISSN $2007-7467$}

(comps.), Aportes de inclusión al conocimiento y alfabetización tecnológica para adultos (pp. 9-24). México: Tabook.

Maldonado, A. y Rodríguez, F. (2016). Innovación en los procesos de enseñanza-aprendizaje: un estudio de casos con la enseñanza justo a tiempo y la instrucción entre pares. Revista Educare, 20(2), 1-21. Doi: http://dx.doi.org/10.15359/ree.20-2.14

Medina, A. (2015). Innovación de la educación y la docencia (2. ${ }^{\mathrm{a}}$ ed.). Madrid: Editorial Centro de Estudios Ramón Areces.

Medina, A. y Domínguez, M. C. (2015). Innovación de la educación y de la docencia. Madrid: Editorial Universitaria Ramón Areces.

Medina, A. y Salvador, F. (2009). Didáctica general. Madrid: Pearson Educación.

Michavila, F. (2009). La innovación educativa. Oportunidades y barreras. Revista de Ciencia, Pensamiento y Cultura, 185, 3-8. Doi: 10.3989/arbor.2009.extran1201

Miranda, L., Angulo, L. y Román, G. (2018). El programa perfiles, dinámicas y desafíos de la educación costarricense: una propuesta para la innovación pedagógica y la producción de materiales y recursos didácticos tecnológicos en la Universidad Nacional, Costa Rica. Revista Educare, 22(1), 1-24. Doi: http://dx.doi.org/10.15359/ree.22-1.10

Organización de las Naciones Unidas [ONU] (2016). Innovación educativa. Recuperado de http://unesdoc.unesco.org/images/0024/002470/247005s.pdf

Organización de las Naciones Unidas para la Educación, la Ciencia y la Cultura [UNESCO] (2015). Estrategia de educación en la UNESCO 2014-2021. Recuperado de https://unesdoc.unesco.org/ark:/48223/pf0000231288_spa.

Ortega, P., Ramírez, M., Torres, J., López, A., Servín, C., Suárez, L. y Ruiz, B. (2007). Modelo de innovación educativa. Un marco para la formación y el desarrollo de una cultura de la innovación. Ried. Revista Iberoamericana de Educación a Distancia, 10(1), 145-173. Recuperado de http://www.redalyc.org/comocitar.oa?id=331427206010. 


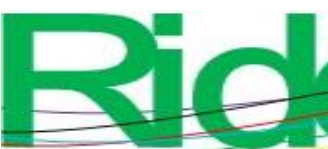

Revista Iberoamericana para la Investigación y el Desarrollo Educativo

ISSN $2007-7467$

Parra, H., Tobón, S. y López, J. (2015). Docencia socioformativa y desempeño académico en la educación superior. Paradigma, 36(1), 42-55. Recuperado de http://revistas.upel.edu.ve/index.php/paradigma/article/view/2653.

Porto, A. y Mosteiro, M. (2014). Innovación y calidad en la formación del profesorado universitario. Revista Electrónica Interuniversitaria de Formación del Profesorado, 17(3), 141-156.

Rodríguez, M., González, E. y Gámiz, V. (2016). La perspectiva de innovación que se impulsa desde la educación superior. Journal for Educators, Teachers and Trainers, 7(1), 193-209.

Secretaría de Educación Pública [SEP] (2013). Las estrategias y los instrumentos de evaluación desde el enfoque formativo. México: SEP.

Strauss, A. y Corbin, J. (2002). Bases de la investigación cualitativa. Técnicas y procedimientos para desarrollar la teoría fundamentada. Bogotá-Colombia: CONTUS. Editorial Universidad de Antioquia.

Tobón, S. (2014). Proyectos formativos: teoría y práctica. México: Pearson.

Vivar, C., Arantzamendi, M., López, O. y Gordo, C. (2010). La teoría fundamentada como metodología de investigación cualitativa en enfermería. Index de Enfermería, 19(4), 283-288. Recuperado de http://scielo.isciii.es/scielo.php?script=sci_arttext\&pid=S113212962010000300011. 


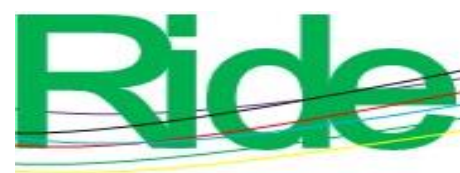

Revista Iberoamericana para la Investigación y el Desarrollo Educativo ISSN 2007 - 7467

\begin{tabular}{|c|c|}
\hline Rol de Contribución & Autor (es) \\
\hline Conceptualización & Mario Alberto Vázquez García \\
\hline Metodología & Isolina Gonzalez Castro \\
\hline Investigación & Martha Alejandrina Zavala Guirado. \\
\hline $\begin{array}{l}\text { Escritura - Preparación del borrador } \\
\text { original }\end{array}$ & Isolina Gonzalez Castro \\
\hline Escritura - Revisión y edición & Mario Alberto Vázquez García \\
\hline Visualización & Martha Alejandrina Zavala Guirado. \\
\hline Supervisión & Martha Alejandrina Zavala Guirado. \\
\hline Administración de Proyectos & Martha Alejandrina Zavala Guirado. \\
\hline Adquisición de fondos & Martha Alejandrina Zavala Guirado. \\
\hline
\end{tabular}

